\section{Eating disorders in Asian girls}

SIR: We would like to comment on eating disorders in Asian girls in the light of two recent papers by Mumford et al (Journal, February 1991, 158, 222228) and Bryant-Waugh \& Lask (Journal, February 1991, 158, 229-233).

Mumford et al, studying Asian schoolgirls with bulimia nervosa whose mean age was 15.1 years, found that symptoms correlated with 'traditionalism' within the family. Drs Bryant-Waugh \& Lask were the first to describe anorexia nervosa in Asian children. They found a younger group than did the former authors, with a mean age of onset of 12.6 years. These children came from families stressed by isolation, poor marital relationships and cultural conflicts.

In our own retrospective study of the Nottingham case register between 1976 and 1984, 120 cases of anorexia nervosa and 46 cases of bulimia nervosa were identified using ICD-10 (World Health Organization, 1986) and DSM-III-R criteria. The annual incidence of anorexia nervosa in Nottingham during the study period ranged from 2.3 to 4.1 per 100000 total population. The annual incidence of bulimia nervosa was reported by Russell (1979) to be 0.003 cases per 100000 when he first described the condition; this had risen to 2.84 per 100000 in 1984.

We found only four cases of anorexia nervosa in Asian girls: one was a prepubertal girl aged 12 years diagnosed in 1983; the other three were aged 16, 17 and 19 years and were diagnosed in 1984, 1980 and 1979 respectively. The younger girls (aged 12 and 16) had problems originating from family distress, especially poor marital relationships, unemployment, isolation, and social problems in extended families. These patients correspond closely to the group described by Drs Bryant-Waugh \& Lask. The problems of the two older girls (aged 17 and 19) related to cultural clashes, including emancipation of women, and had features in common with the bulimia nervosa group described by Mumford et al. We failed to identify any Asian girls with bulimia nervosa, but our study ended with cases identified in 1984.

It seems that the aetiological background in the development of eating disorders in Asian girls may vary in younger and older groups, younger girls becoming ill as a result of family difficulties, and older girls when traditional family attitudes clash with more personal Westernised ones, especially over 'female role' and marriage.

The collection of more detailed data about ethnicity in the 1991 Census, together with a heightened awareness of eating disorders in Asian girls, will allow, for the first time, accurate measurement of population incidence. Examination of different cohorts may well highlight changes reflecting increasing Westernișation.

RusselL, G. F. M. (1979) Bulimia nervosa: an ominous variant of anorexia nervosa. Psychological Medicine, 9, 429-448.

World Health Organization (1986) Draft of Chapter V. Geneva: WHO.

\section{P. BENDALL \\ M. HAMILTON \\ N. HoldeN}

Department of Psychiatry

\section{Mapperley Hospital \\ Porchester Road \\ Nottingham NG3 6AA}

\section{Motor disorder in severe mental handicap}

SIR: Rogers et al (Journal, January 1991, 158, 97-102) find that motor disorder is significantly associated with more severe mental handicap, suggesting greater cerebral dysfunction, and thus a connection between motor and cerebral disorder. This therefore suggests that further investigation of those with more severe handicap would be of value in an attempt to elucidate the pathophysiology of movement disorders.

Kohen (Journal, October 1990, 157, 621), and Mathew et al (Journal, April 1991, 158, 571) referring to dyskinesia in people with mental handicap, suggest the use of DISCUS (Sprague et al, 1989) as a rating scale standardised on people with a mental handicap. This is a welcome reference to a scale that has been infrequently used.

However, there are problems reported in the use of the DISCUS, particularly in the assessment of those with severe and profound handicap - mainly regarding co-operation in the examination of the lingual area. While copious information on this difficult group is given in Sprague et al (1984) using the DISCo (the DISCUS predecessor), Sprague et al (1989), using the DISCUS itself, reported $73.9 \%$ of the patient group with an unspecified level of handicap. Granger et al (1987) used DISCUS and found full co-operation in 121 of 306 patients in the severe/ profound group compared with Sprague's 34 of 99 patients using DIS-Co.

Another reservation in the use of the DISCUS is that items such as teeth grinding are usually considered 'non-dyskinesia related' and are not scored. The DISCUS is designed to quantify and monitor tardive dyskinesia, and Sprague et al (1989), in their training workshops, teach participants how to separate self stimulation from dyskinesia. Dr Rogers seeks specifically to avoid such judgements in his 
descriptive study. An inclusive approach would seem particularly appropriate for those with severe handicap where the aetiology of repetitive movements is speculative.

Therefore the descriptive categories of the DISCo or DISCUS can be appropriately employed, provided that attention is adequately paid to cooperation levels and their influence on study results. It must also be recognised that other movements, while not regarded as forming part of a tardive dyskinesia spectrum, represent aspects of motor disorder and are relevant. On this basis, a Nottingham group is currently examining abnormal movements in people with severe impairments.

Granger, D. A., Yurkunski, J. M., Miller, N. H., et al (1987) Systematic dyskinesia examination of profoundly mentally retarded persons: co-operation and assessment. American Journal of Mental Deficiency, 92, 155-160.

Sprague, R. L., KAlachini,, J. E., Breuning, S. E., et al (1984) The dyskinesia identification system-Coldwater (DIS-Co): A tardive dyskinesia rating scale for the developmentally disabled. Psychopharmacology Bulletin, 20, 328-338.

- — SHAw, K. M. (1989) Psychometric properties of the dyskinesia identification system: condensed user scale (DISCUS). Mental Retardation, 27, 141-148.

Department of Mental Handicap

JO JONES

Highbury Hospital

Bulwell

Nottingham NG6 9DR

\section{Delusional jealousy in paranoid disorders}

SIR: Soyka et al (Journal, April 1991, 158, 549-553) examined the prevalence of delusional jealousy in various psychiatric disorders and reported $6.7 \%$ in paranoid disorders. This might be an underestimate because of the low admission rates of patients in whom delusional jealousy is the only symptom of their illness, unlike patients with other psychotic syndromes. The authors themselves had highlighted the reluctance on the part of patients, and their spouses, to talk about this particular symptom, sometimes leading to difficulty in diagnosis. One would naturally expect more reluctance in treatment acceptance, especially when it comes to admission to psychiatric hospital. Thus by studying only in-patients, the authors could not exclude the possibility of selection bias affecting the prevalence of delusional jealousy.

We reviewed the case records of 297 patients diagnosed to have a paranoid disorder (ICD-9; World Health Organization, 1978) who presented to our department over a 10-year period (1979-1989). Of the 93 patients who received a diagnosis of a paranoid disorder, delusional jealousy was documented in 15 individuals, giving the symptom a prevalence of $16 \%$. Among the 16 patients with paranoid disorders who received in-patient treatment, only one individual had delusions of jealousy. In our series, the prevalence of this symptom among in-patients is similar to that of Soyka et al. Thus, our findings show marked differences in the prevalence of the symptom dependent on the admission status, with a higher figure for out-patients than among those admitted to the wards.

When DSM-III-R criteria were applied to these case records, only 60 individuals met the criteria for delusional disorder. Interestingly, all the patients with delusions of jealousy met the criteria, thus increasing the prevalence of delusional jealousy to $25 \%$ among patients with delusional disorder. This suggests that the actual prevalence of delusional jealousy could be higher with the use of more restrictive criteria. However, the prevalence of this symptom in the community would probably be different and would be difficult to document.

World Health Organization (1978) Mental Disorders: Glossary and Guide to their Classification in accordance with the minth revision of the International Classification of Diseases (ICD-9). Geneva: WHO.

Department of Psychiatry

K. S. SHAJ

Christian Medical College

Vellore 632002

India

SIR: We read with interest Soyka et al's study (Journal, April 1991, 158, 549-553) of the prevalence of delusional jealousy, and would like to offer the following comments.

Firstly, delusional jealousy involves the lover, marital or sexual partner and hence occurs in those who have or have ever had one. Therefore, considering the whole patient population, ignoring the marital or sexual status and history, and including those who never had a lover or partner, as done in this study, would give erroneously low figures.

Secondly, the authors suggest that the difference in prevalence of delusional jealousy in affective disorders $(0.1 \%)$ and schizophrenia $(2.5 \%)$ may be of value in the differential diagnosis of the two disorders. This opinion is rather far fetched in view of the fact that among those with delusional jealousy $3-16 \%$ had depressive illness, while $17-44 \%$ had schizophrenia (Gelder et al, 1989).

Finally, this study supports the surprising earlier observations (Gelder et al, 1989) of 'delusional' jealousy occurring in 'neuroses'. 